\title{
Recent advanced applications and services for intelligent ubiquitous environments
}

\author{
Jongsung Kim • Han-Chieh Chao • \\ Uyen Trang Nguyen
}

Published online: 18 September 2014

(C) Springer Science+Business Media New York 2014

Intelligent ubiquitous environments (IUEs) are a new paradigm that offers computing and communication services at any time and anywhere. IUEs are normally implemented by advanced applications and services including cloud computing and mobile computing. The services computing is a new cross-discipline that covers the science and technology needed to bridge the gap between business services and IT/telecommunication services. The goal of this special issue is to develop new computing technology and thereby enables more advanced IT/telecommunication services to support business services more efficiently and effectively. This special issue aims to foster the dissemination of high quality research in any new theory, technique, and research related to recent advanced services computing technology for IUEs, as well as to enhance its state-of-the-art.

Topics of primary interest include:

- Application integration services

- Business performance management

- Social networked applications and services

\footnotetext{
J. $\operatorname{Kim}(\varangle)$

Department of Mathematics \& Department of Financial Information Security,

Kookmin University, 77, Jeongneung-Ro, Seongbuk-Gu, Seoul 136-702, Republic of Korea e-mail: jongsung.k@gmail.com

H.-C. Chao

Institute of Computer Science \& Information and Department of Electronic Engineering, National Ilan University, I-Lan, Taiwan

e-mail: hcc@niu.edu.tw

U. T. Nguyen

Department of Computer Science and Engineering, York University, 4700 Keele Street, Toronto, ON M3J 1P3, Canada e-mail: utn@cse.yorku.ca
} 
- E-business solutions

- Linkage between IT services and business services

- Security, privacy and trust in business process management

- Service computing process modeling, transformation and integration

- Software architecture design, development and deployment

- Cloud computing

- Resource acquisition models in cloud/utility computing

- Security management, analysis for cloud/utility services

- Utility business services

- Utility computing

- Web computing

- Web service management and collaboration

- Web service security and privacy

- Service-oriented business consulting methodologies and utilities

- Services modeling

- Human interface for mobile/ubiquitous/pervasive environments

- Mobile/ubiquitous/pervasive quality of service (QoS) adaptation

- Software-defined radio, reconfigurable radio network

- Service-oriented architecture (SOA)

- Telecommunication Services

- Service-oriented Telco architectures and business processes

- IP Multimedia Subsystem (IMS)

- Next generation networks

We received 56 manuscripts in total. Each manuscript was blindly reviewed by at least three reviewers consisting of guest editors and external reviewers. After the review process, nine manuscripts were finally selected for this Special Issue.

The selected nine manuscripts are summarized as follows: The first manuscript is titled, Design of Online Survey System with an Advanced IPA Discrimination Index for Customer Satisfaction Assessment. It is authored by Hsiang-Chuan Liu, Bai-Cheng Jeng, Yi-Ting Mai, Yu-Du Jheng, Hao-Tung Lin. To analyze customer satisfaction, importance-performance analysis (IPA) and Parasuraman, Zeithaml \& Berry (PZB) are just some of the enhanced analysis tools that measure user satisfaction. This paper introduces a novel web-based user feedback survey system with a proposed new IPA index. To identify customer satisfaction, the new IPA model can reveal a more accurate quantity comparison and find potentially helpful information for customer satisfaction.

The second manuscript is titled, Quality evaluation and best service choice for cloud computing based on user preference and weights of attributes using the Analytic Network Process. It is authored by Cheol-Rim Choi, Hwa-Young Jeong. The paper proposes a system that calculates the priority weights for each quality attribute according to the quality preference of a user and the interrelation analysis results between the attributes, and reflects the weights in selecting the cloud computing service. Through the proposed system, users can easily perform the process of calculating the weights and selecting the best services according to their quality preference. The simulation results show the effectiveness of the system. 
The third manuscript is titled, Lightweight Secure Storage Model with FaultTolerance in Cloud Environment. It is authored by Mahra Ahmed, Quang Hieu Vu, Rasool Asal, Hassan Al-Muhairi, Chan Yeob Yeun. According to several surveys, data security is the main reason for people's reluctance to employ cloud computing. In this paper, the authors address the concern of data security by introducing SECRESO, a SEcure storage model for Cloud data based on Reed-Solomon code. In the proposed model, one can enhance Reed-Solomon code with an extra security layer in which a password is always required to reconstruct the data. Additionally, to support fault tolerance, the paper also introduces a log based data recovery scheme that allows data recovery even if the password is unknown.

The fourth manuscript is titled, Public IT Service Strategy for Social Information Security in the Intelligence All-Things Environment. It is authored by Jinho Yoo, Hangbae Chang. This paper proposes ways to improve public information service for the social information security of individuals such as personal or policy information that are dealt with in public information service.

The fifth manuscript is titled, An Enhanced Smartphone Security Model based on Information Security Management System (ISMS). It is authored by Jong Hyuk Park, Ki Jung Yi, Young-Sik Jeong. A Smartphone-ISMS model based on ISMS (Information Security Management System) is proposed in this paper. Firstly, this paper defines the elements of smartphone security threats, which can occur in the smartphone environment, and the requirements for smartphone security. Based on the results, this work derives seven relevant control items by combining existing ISMSbased information security models with the smartphone environment.

The sixth manuscript is titled, An Efficient Novel Online Shopping Mechanism based on Quantum Communication. It is authored by Yao-Hsin Chou, Fang-Jhu Lin, Guo-Jyun Zeng. The paper proposes a quantum secure direct communication protocol, and applies it to online shopping system to raise a novel online trading mechanism. This quantum-based trading system is more secure than traditional ones. Compared to other quantum protocols, its cost is lower and the implementation is more practical than others.

The seventh manuscript is titled, Correlating Messages from Multiple IM Networks to Identify Digital Forensic Artifacts. It is authored Muhammmad Yasin, Firdous Kausar, Eisa Aleisa, Jongsung Kim. This paper focuses on a unique case in which two group members of criminal network are communicating through IM aggregator (like Digsby) and using multiple IM protocols to complete a single conversation session instead of following a traditional single IM client such as Yahoo Messenger for whole conversation. They propose a method to identify that multiple IM protocols are used for single conversation session and describe how to establish a sequence of collected messages. An analysis of volatile memory is performed to collect the remnants of whole or partial conversation, as supportive or actual evidence.

The eighth manuscript is titled, IoT sensing framework with inter-cloud computing capability in vehicular networking. It is authored by Jiafu Wan, Caifeng Zou, Keliang Zhou, Rongshuang Lu, Di Li. The paper proposes a novel business model based on platform production services (PPS), and IoT sensing framework in vehicular networking. The paper also designs an inter-cloud computing architecture for vehicular networking applications (VNA), and builds its semantic modeling analysis. Then 
they present a vehicle maintenance services (VMS) event processing flow in VNA. The performance evaluation results show that the efficiency of the proposed scheme increases.

The last manuscript is titled, $P S O$ based time series models applied in exchange rate forecasting for business performance management. It is authored by Jui-Fang Chang, Yueh-Min Huang. This paper uses the PSO algorithm to develop three new models, PSOGARCH, PSOEGARCH, and PSOGJR-GARCH, for improving business performance management. The forecasting outcomes of traditional models are compared to those of PSOGARCH, PSOEGARCH, and PSOGJR-GARCH models. In the end, the result show PSOGJR-GARCH model had the best forecasting ability.

Finally, we would like to thank all authors for their contributions to this special issue. We also extend our thanks to the external reviewers for their excellent job in reviewing the manuscripts.

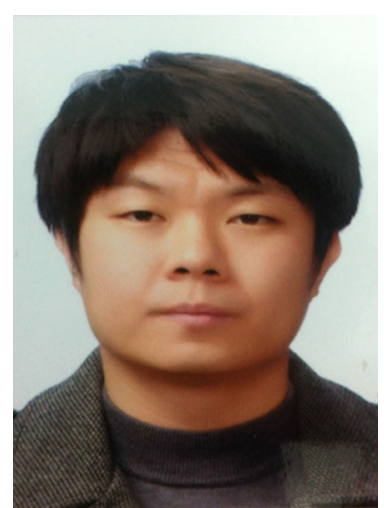

Jongsung Kim received his Bachelor and Master degrees in Mathematics from Korea university, Korea in 2000 and 2002, respectively. He received double Doctoral degrees completed in November 2006 and February 2007 at the ESAT/COSIC group Katholieke Universiteit Leuven and at Engineering in Information Security of Korea University, respectively. He had been a Research Professor of Center for Information Security Technologies (CIST) at Korea University, Korea, from March 2007 till August 2009, and an assistant professor of department of e-business at Kyungnam University, Korea, from September 2009 till February 2013. Dr. Kim has been an assistant professor of department of mathematics at Kookmin University, Korea, since March 2013. Dr. Kim has published about 60 research papers in international journals and conferences. He has been serving as chairs, program committee, or organizing committee chair for many international conferences and workshops. He is editorial board member of International Journal of Information Technology, Communications and Convergence (IJITCC), International Journal of Communication Networks and Distributed Systems (IJCNDS), InderScience and Journal of Convergence (JoC), FTRA Publishing, and Humancentric Computing and Information Sciences (HCIS), Springer. In addition, he has been serving as a Guest Editor for international journals by some publishers: Springer, Elsevier, John Wiley, Oxford Univ. press, Inderscience. His research interests include security issues, cryptography, ubiquitous computing systems and digital forensics.

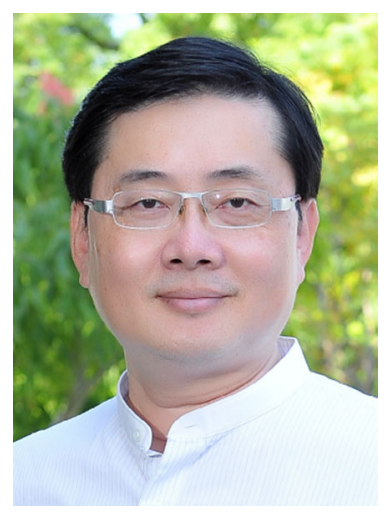

Han-Chieh Chao is a joint appointed Full Professor of the Department Computer Science \& Information Engineering and Electronic Engineering of National Ilan University, I-Lan, Taiwan (NIU). He is serving as the President since August 2010 for NIU as well. He was the Director of the Computer Center for Ministry of Education Taiwan from September 2008 to July 2010. His research interests include High Speed Networks, Wireless Networks, IPv6 based Networks, Digital Creative Arts, e-Government and Digital Divide. He received his MS and Ph.D. degrees in Electrical Engineering from Purdue University in 1989 and 1993 respectively. He has authored or co-authored 4 books and has published about 400 refereed professional research papers. He has completed more than 100 MSEE thesis students and 4 Ph.D. students. Dr. Chao has been invited frequently to give talks at national and international conferences and research organizations. Dr. Chao is the Editor-in-Chief for IET Networks, Journal of Internet Technology, International Journal of Internet Protocol 
Technology and International Journal of Ad Hoc and Ubiquitous Computing. Dr. Chao has served as the guest editors for Mobile Networking and Applications (ACM MONET), IEEE JSAC, IEEE Communications Magazine, IEEE Systems Journal, Computer Communications, IEE Proceedings Communications, the Computer Journal, Telecommunication Systems, Wireless Personal Communications, and Wireless Communications \& Mobile Computing. Dr. Chao is an IEEE senior member and a Fellow of IET (IEE).

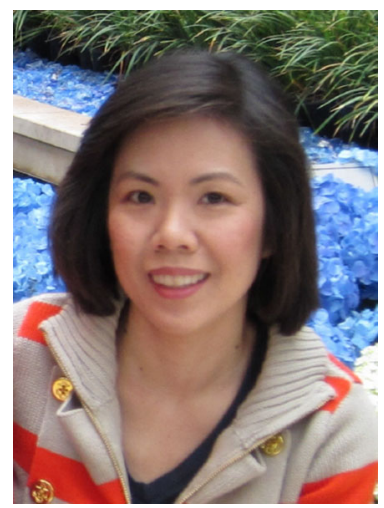

Uyen Trang Nguyen received her Bachelor of Computer Science and Master of Computer Science degrees in 1993 and 1997, respectively, from Concordia University, Montreal, Canada. She completed her Ph.D. degree at the University of Toronto, Canada, in 2003. From 1995 to 1997 she was a software engineer at Nortel Networks, Montreal, Canada. She joined the Department of Computer Science and Engineering at York University, Toronto, Canada, in 2002 and is currently an Associate Professor. Her research interests are in the areas of mobile and ubiquitous computing, wireless networking, multimedia applications and network security. Prof. Nguyen has published more than 40 papers in refereed international journals and conference and workshop proceedings. She is on the editorial board of Communications Journal published by ACTA Press. She is serving as a program co-chair for the 3rd International Conference on Mobile, Ubiquitous, and Intelligent Computing (MUSIC 2012). She is also a program vice-chair of the 13th IEEE International Conference on High

Performance Computing and Communications (HPCC 2011) and the 7th International Conference on Future Information Technology (FutureTech 2012). 